\title{
Late-time behavior of fast neutrino oscillations
}

\author{
Soumya Bhattacharyya $\odot^{*}$ and Basudeb Dasgupta $\odot^{\dagger}$ \\ Tata Institute of Fundamental Research, Homi Bhabha Road, Mumbai 400005, India
}

(Received 21 July 2020; accepted 17 August 2020; published 16 September 2020)

\begin{abstract}
We study the fully nonlinear fast flavor evolution of neutrinos in $1+1$ dimensions. Our numerical analysis shows that at late times, the system reaches an approximately steady state. Using the steady-state approximation, we analytically show that the spatial variation of the polarization vectors is given by their precession around a common axis, which itself has a motion reminiscent of a gyroscopic pendulum. We then show that the steady-state solution to the equations of motion cannot be separated in position and velocity - that is, the motion is not collective in the usual sense. However, the fast evolution allows spectral-swap-like dynamics leading to partial decoherence over a range of velocities, constrained by the conservation of lepton number(s). Finally, we numerically show that at late times, the transverse components of the polarization vectors become randomly oriented at different spatial locations for any velocity mode and lepton asymmetry.
\end{abstract}

DOI: 10.1103/PhysRevD.102.063018

\section{INTRODUCTION}

Neutrinos emitted by stars present valuable opportunities to study neutrino properties [1]. While solar neutrinos have famously helped zero in on the large mixing angle scenario, neutrinos from supernovae may yet provide a unique opportunity to study neutrino-neutrino interactions-a crucial piece of the standard model of particle physics that has not been tested directly.

The rate of neutrino oscillations is typically dictated by the vacuum oscillation frequency, $\omega$, and the matter potential, $\lambda$ [2-4]. Until the early 2000s, it was believed that this paradigm was sufficient to describe neutrino oscillations inside supernovae as well [5]. At that time, the outstanding problem of the field appeared to be to understand the effect of fluctuations in the background matter density [6-8].

Following the pioneering papers by Pantaleone $[9,10]$, however, it became clear that the issue is more subtle $[11,12]$. Owing to the large neutrino density, even freestreaming neutrinos experience significant forwardscattering off other neutrinos. Such scattering leads to a self-interaction potential, $\mu \gg \omega$, that is proportional to the neutrino density and can dominate over the vacuum term.

\footnotetext{
*soumya.bhattacharyya@theory.tifr.res.in

†bdasgupta@theory.tifr.res.in
}

Published by the American Physical Society under the terms of the Creative Commons Attribution 4.0 International license. Further distribution of this work must maintain attribution to the author(s) and the published article's title, journal citation, and DOI. Funded by SCOAP ${ }^{3}$.
As a result, a gamut of new collective flavor transformations can occur inside supernovae.

The so-called "slow" collective effects, with an intrinsic rate $\sim \sqrt{\omega \mu}$, are already faster than usual neutrino oscillations. These lead to a variety of new phenomena: e.g., synchronization [11], bipolar oscillations [12-16], spectral swaps [17-20], three-flavor effects [21-24], multiangle effects [25-28], decoherence [29-32], and linear instabilities [33], including those that break symmetries of direction [34,35], space [36,37], and time [38,39]. Related developments that followed the influential papers by Duan, Fuller, Carslon and Qian, and their phenomenological consequences have been reviewed in Refs. [40-43]; see also references therein.

Ray Sawyer pointed out that much more rapid "fast" flavor conversions can take place [44-47]. These have a frequency $\sim \mu$ and might have a much more drastic effect for neutrino physics [48-68] as well as supernova astrophysics [69-75]. The criterion for fast conversions to occur appears to be related to that for slow conversions-i.e., the difference of neutrino and antineutrino flux distributions in the momentum space must have a zero crossing [20], though a more detailed understanding still remains wanting.

The flavor evolution of a dense neutrino gas is governed by a large number of coupled nonlinear partial differential equations. These are almost always very difficult to solve. Although linearized stability analysis is useful to ascertain if and when fast conversion takes place, it cannot directly answer an important question: What is the impact of fast flavor conversion on observable neutrino fluxes or the explosion mechanism? This is a significantly harder problem that requires understanding the nature of the solution in the nonlinear regime. A step in this direction was taken by 
Sen and one of the present authors [51], where the flavor evolution of a 4-beam model in $0+1$ dimensions was understood in the fully nonlinear regime.

In this work, we take another step in the same direction. We consider a dense neutrino gas in $1+1$ dimensions, with a spectrum of velocity modes, and analyze the coupled flavor evolution of the neutrino system into the nonlinear regime. Our numerical analysis suggests that the system reaches an approximately steady state at late time. In the steady-state approximation, we analytically show that the spatial variation of the polarization vectors is given by their precession around a gyrating flavor pendulum with a fixed length, spin, and energy, and the solution is not collective. The polarization vectors, when averaged over space, however, exhibit complete (partial) decoherence for zero (nonzero) lepton asymmetry. For partial decoherence, the nonvanishing range of velocity modes is dictated by the conservation of lepton numbers. This kinematic decoherence stems from randomization of the transverse components. Numerical examples confirm these analytical insights.

The paper is structured as follows: Section II recollects the equations of motion, followed by our analytical results on the nature of the solution, conserved quantities, partial decoherence, and its dependence on lepton asymmetry. Section III has our numerical results and their comparison with analytical claims. We conclude in Sec. IV.

\section{ANALYTICAL RESULTS}

\section{A. Equations of motion}

Neglecting momentum-changing collisions, the space and time evolution of two flavors of neutrinos with velocity $\mathbf{v}$ and vacuum oscillation frequency $\omega=\left|\Delta m^{2}\right| /(2 \mathcal{E})$ is given by $[46,47]$

$$
\left(\partial_{t}+\mathbf{v} \cdot \nabla_{\mathbf{x}}\right) \mathbf{P}_{\omega, \mathbf{v}}(\mathbf{x}, t)=\mathbf{H}_{\omega, \mathbf{v}}(\mathbf{x}, t) \times \mathbf{P}_{\omega, \mathbf{v}}(\mathbf{x}, t) .
$$

Here $\mathbf{P}_{\omega, \mathbf{v}}=g_{\omega, \mathbf{v}} \mathbf{S}_{\omega, \mathbf{v}}=g_{\omega, \mathbf{v}}\left(s_{v}^{(1)}, s_{v}^{(2)}, s_{v}^{(3)}\right)^{T}$ is a so-called polarization vector, that encapsulates the flavor composition of the neutrino mode $\omega$, v, while $\mathbf{H}_{\omega, \mathbf{v}}=\mathbf{H}_{\omega}^{\mathrm{vac}}+\mathbf{H}^{\mathrm{mat}}+$ $\mathbf{H}_{\omega, \mathbf{v}}^{\text {self }}$ is the Bloch vector representation of the flavorevolution Hamiltonian for each mode. The vector $\mathbf{H}_{\omega}^{\mathrm{vac}}=$ $\omega \mathbf{B}$ encodes vacuum oscillations with $\mathbf{B}=(\sin 2 \theta, 0, \cos 2 \theta)^{T}$; the vector $\mathbf{H}^{\text {mat }}=\lambda \mathbf{L}$ encodes the matter effects with $\lambda=\sqrt{2} G_{F}\left(n_{e^{-}}-n_{e^{+}}\right)$and $\mathbf{L}=(0,0,1)^{T} ;$ and $\mathbf{H}_{\omega, \mathbf{v}}^{\text {self }}=$ $\mu \int d \Gamma \prime(1-\mathbf{v} . \mathbf{v} \prime) \mathbf{P}_{\omega \prime, \mathbf{v}}(x, t)$ encodes the self-interactions, with $\mu$ being the $\nu-\nu$ potential. Note that we work in a basis $\left\{\hat{\mathbf{e}}_{1}, \hat{\mathbf{e}}_{2}, \hat{\mathbf{e}}_{3}\right\}$, where the Bloch vector for a $\nu_{e}$ points along the direction $\hat{\mathbf{e}}_{3}$.

In the fast flavor limit, the vacuum and matter term in Eq. (1) are negligible compared to the neutrino potential term, so the solution for $\mathbf{P}_{\omega, \mathbf{v}}$ becomes $\omega$ independent. The self-term then enters the Hamiltonian only through the electron lepton number (ELN) distribution-i.e., the difference of occupation number densities integrated over energy, defined by $G_{\mathbf{v}}=\int d \omega g_{\omega, \mathbf{v}}$-thereby allowing us to rewrite Eq. (1) in $1+1 \mathrm{D}$ as

$$
\left(\partial_{t}+v \partial_{x}\right) \mathbf{P}_{v}(x, t)=\int_{-1}^{1} d v^{\prime}\left(1-v v^{\prime}\right) \mathbf{P}_{v^{\prime}}(x, t) \times \mathbf{P}_{v}(x, t),
$$

where $\quad \mathbf{P}_{v}(x, t)=G_{v} \mathbf{S}_{v}(x, t)=G_{v}\left(s_{v}^{(1)}, s_{v}^{(2)}, s_{v}^{(3)}\right)^{T}$. We choose our units in Eq. (2) such that the neutrino selfinteraction potential $\mu$ is 1 , with length and time expressed in units of $\mu^{-1}$. $G_{v}$ encodes the amount of lepton asymmetry of the system as a function of velocity modes.

One can obtain some more insight into the equations of motion by expanding $\mathbf{P}_{v}(x, t)$ for each velocity mode in terms of Legendre polynomials, $L_{n}(v)$. Using the expansion $\mathbf{P}_{v}(x, t)=\sum_{n=0}^{\infty}\left(n+\frac{1}{2}\right) \mathbf{M}_{n}(x, t) L_{n}(v)$, and using the orthogonal property of Legendre polynomials, i.e., $\int_{-1}^{1} L_{r}(v) L_{n}(v) d v=2 \delta_{r n} /(2 r+1)$, by following Ref. [29], one can rewrite Eq. (2) as

$\left(\partial_{t}+v \partial_{x}\right) \mathbf{P}_{v}(x, t)=\left(\mathbf{M}_{0}(x, t)-v \mathbf{M}_{1}(x, t)\right) \times \mathbf{P}_{v}(x, t)$.

\section{B. Steady state}

We conjecture that at late times the system becomes approximately stationary in time, which says that in Eq. (2) we can drop the $t$ dependence from all the quantities. We will verify this conjecture in our numerical survey described in Sec. III.

In the steady state, one can write Eq. (2) as

$$
d_{x} \mathbf{P}_{v}(x)=\left(\frac{\mathbf{M}_{0}(x)}{v}-\mathbf{M}_{1}(x)\right) \times \mathbf{P}_{v}(x)
$$

and the equation for each multipole moment $\mathbf{M}_{r}(x)$ as

$d_{x} \mathbf{M}_{r}(x)=\mathbf{M}_{0}(x) \times \sum_{n=0}^{\infty} \ell_{r n} \mathbf{M}_{n}(x)-\mathbf{M}_{1}(x) \times \mathbf{M}_{r}(x)$,

where

$$
\ell_{r n}=\left(n+\frac{1}{2}\right) \int_{-1}^{1} \frac{L_{n}(v) L_{r}(v)}{v} d v
$$

From Eq. (5), for $r=1$, and using $\ell_{1 n}=(2 n+1) \delta_{0 n}$, one gets that $\mathbf{M}_{1}(x)$ is constant in space. This means that all the polarization vectors of different velocity modes precess about a fixed axis $\mathbf{M}_{1}$ with the same frequency. This common motion can be hidden away by considering a rotating frame, using the transformation

$$
\widetilde{d}_{x} \widetilde{\mathbf{P}}_{v}(x)=d_{x} \mathbf{P}_{v}(x)+\mathbf{M}_{1} \times \mathbf{P}_{v}(x),
$$


where we introduce the notation that all the quantities in the rotating frame are denoted by a tilde, with spatial derivatives in the rotating frame as $\widetilde{d}_{x}$, etc.

Using the rotation formula described in Eq. (7), the equations of motion for the system at late times, described by Eqs. (4) and (5) in the rotating frame, look like

$$
\begin{aligned}
& \widetilde{d}_{x} \widetilde{\mathbf{P}}_{v}(x)=\frac{\widetilde{\mathbf{M}}_{0}(x)}{v} \times \widetilde{\mathbf{P}}_{v}(x), \\
& \widetilde{d}_{x} \widetilde{\mathbf{M}}_{r}(x)=\widetilde{\mathbf{M}}_{0}(x) \times \sum_{n=0}^{\infty} \ell_{r n} \widetilde{\mathbf{M}}_{n}(x) .
\end{aligned}
$$

Considering $r=0$ and summing both sides of Eq. (8b) with a weightage factor of $\ell_{0 r}$, from $r=0$ to $r=\infty$, one can rewrite Eq. (8b) as two coupled equations:

$$
\begin{aligned}
& \widetilde{d}_{x} \widetilde{\mathbf{M}}_{0}(x)=\widetilde{\mathbf{D}}(x) \times \widetilde{\mathbf{M}}_{0}(x), \\
& \widetilde{d}_{x} \widetilde{\mathbf{D}}(x)=\widetilde{\mathbf{B}}(x) \times \widetilde{\mathbf{M}}_{0}(x),
\end{aligned}
$$

where

$$
\begin{gathered}
\widetilde{\mathbf{D}}(\mathbf{x})=-\sum_{n=0}^{\infty} \ell_{0 n} \widetilde{\mathbf{M}}_{n}(x), \\
\widetilde{\mathbf{B}}(x)=\sum_{r, n=0}^{\infty} \ell_{0 r} \ell_{r n} \widetilde{\mathbf{M}}_{n}(x) .
\end{gathered}
$$

Similarly, by summing both sides of Eq. (8b), with a weightage factor of $\sum_{p=0}^{\infty} \ell_{0 p} \ell_{p r}$, from $r=0$ to $r=\infty$, one obtains an equation for $\widetilde{\mathbf{B}}(x)$ :

$$
\widetilde{d}_{x} \widetilde{\mathbf{B}}(x)=\sum_{p, r, n=0}^{\infty} \ell_{0 p} \ell_{p r} \ell_{r n}\left(\widetilde{\mathbf{M}}_{0}(x) \times \widetilde{\mathbf{M}}_{n}(x)\right) .
$$

Equations (9a) and (9b) are nominally the same set of coupled equations which describe the "gyroscopic pendulum" (see Ref. [15]), with the difference that $\widetilde{\mathbf{B}}(x)$, instead of being a constant, has an equation of motion in space described by Eq. (12). Nevertheless, one can follow Ref. [15] to derive an equation for a gyroscopic pendulum with a position-independent length, spin, and energy. Equation (9a) clearly indicates that $M_{0}$-i.e., the length of $\widetilde{\mathbf{M}}_{0}(x)$, which is the same in rotating and nonrotating frames - is a constant in space. Taking dot products with $\widetilde{\mathbf{D}}(x)$ or $\widetilde{\mathbf{B}}(x)$, respectively, on both sides of Eq. (9a), and with $\widetilde{\mathbf{M}}_{0}(x)$ or $\widetilde{\mathbf{D}}(x)$, respectively, on both sides of Eq. (9b), and with $\widetilde{\mathbf{M}}_{0}(x)$ for Eq. (12), one gets the following set of equations:

$$
\begin{aligned}
& \widetilde{\mathbf{D}}(x) \cdot \widetilde{d}_{x} \widetilde{\mathbf{M}}_{0}(x)=0, \\
& \widetilde{\mathbf{B}}(x) \cdot \widetilde{d}_{x} \widetilde{\mathbf{M}}_{0}(x)=\widetilde{\mathbf{B}}(x) \cdot\left(\widetilde{\mathbf{D}}(x) \times \widetilde{\mathbf{M}}_{0}(x)\right), \\
& \widetilde{\mathbf{M}}_{0}(x) \cdot \widetilde{d}_{x} \widetilde{\mathbf{D}}(x)=0, \\
& \widetilde{\mathbf{D}}(x) \cdot \widetilde{d}_{x} \widetilde{\mathbf{D}}(x)=\widetilde{\mathbf{D}}(x) \cdot\left(\widetilde{\mathbf{B}}(x) \times \widetilde{\mathbf{M}}_{0}(x)\right), \\
& \widetilde{\mathbf{M}}_{0}(x) \cdot \widetilde{d}_{x} \widetilde{\mathbf{B}}(x)=0 .
\end{aligned}
$$

Adding Eqs. (13a) and (13c), one obtains

$$
\widetilde{d}_{x}\left(\widetilde{\mathbf{M}}_{0}(x) \cdot \widetilde{\mathbf{D}}(x)\right)=0
$$

which implies that the "spin"

$$
\widetilde{\mathbf{m}}_{0}(x) \cdot \widetilde{\mathbf{D}}(x)=\sigma=\text { constant }
$$

i.e., it is position independent and, owing to the steady-state approximation, also time independent. Here, $\widetilde{\mathbf{m}}_{0}(x)=$ $\widetilde{\mathbf{M}}_{0}(x) / M_{0}$ is the unit vector along $\widetilde{\mathbf{M}}_{0}(x)$. Similarly, the addition of Eqs. (13b), (13d), and (13e) reveals energy conservation:

$$
\widetilde{d}_{x}\left(\widetilde{\mathbf{B}}(x) \cdot \widetilde{\mathbf{M}}_{0}(x)+\frac{1}{2} \widetilde{\mathbf{D}}(x) \cdot \widetilde{\mathbf{D}}(x)\right)=0,
$$

which implies

$$
\widetilde{\mathbf{B}}(x) \cdot \widetilde{\mathbf{M}}_{0}(x)+\frac{1}{2} \widetilde{\mathbf{D}}(x) \cdot \widetilde{\mathbf{D}}(x)=E=\text { constant } .
$$

Taking a cross product of Eq. (9a) with $\widetilde{\mathbf{M}}_{0}(x)$, one gets

$$
\begin{aligned}
& \widetilde{\mathbf{M}}_{0}(x) \times \widetilde{d}_{x} \widetilde{\mathbf{M}}_{0}(x) \\
& \quad=-\left(\widetilde{\mathbf{M}}_{0}(x) \cdot \widetilde{\mathbf{D}}(x)\right) \widetilde{\mathbf{M}}_{0}(x)+M_{0}^{2} \widetilde{\mathbf{D}}(x) .
\end{aligned}
$$

Dividing both sides of Eq. (18) by $M_{0}^{2}$ and then using Eq. (15), one can rewrite Eq. (18) as

$$
\widetilde{\mathbf{D}}(x)=\widetilde{\mathbf{m}}_{0}(x) \times \widetilde{d}_{x} \widetilde{\mathbf{m}}_{0}(x)+\sigma \widetilde{\mathbf{m}}_{0}(x) .
$$

Differentiating Eq. (19) once, and using the spatial conservation of $\sigma$ along with Eq. (9b), gives

$\widetilde{\mathbf{m}}_{0}(x) \times \widetilde{d}_{x}^{2} \widetilde{\mathbf{m}}_{0}(x)+\sigma \widetilde{d}_{x} \widetilde{\mathbf{m}}_{0}(x)=M_{0} \widetilde{\mathbf{B}}(x) \times \widetilde{\mathbf{m}}_{0}(x)$.

The vector $\widetilde{\mathbf{M}}_{0}(x)$ in flavor space plays the role of a gyroscopic pendulum. It has a fixed length at all spatial locations, so that it is restricted to move on a sphere of a fixed radius $M_{0}$. According to Eq. (17), the energy of the pendulum is spatially invariant at late times, where the first term $\widetilde{\mathbf{B}}(x) \cdot \widetilde{\mathbf{M}}_{0}(x)$ is equivalent to the potential energy of 
$\widetilde{\mathbf{M}}_{0}(x)$ in an inhomogeneous magnetic field $\widetilde{\mathbf{B}}(x)$, and $\frac{1}{2} \widetilde{\mathbf{D}}(x) \cdot \widetilde{\mathbf{D}}(x)$ is the rotational energy of the system, with $\widetilde{\mathbf{D}}(x)$ playing the role of the orbital angular momentum of the system. Equation (15) describes one more conserved quantity, $\sigma$, which says that the component of the angular momentum $\widetilde{\mathbf{D}}(x)$ that is parallel to $\widetilde{\mathbf{M}}_{0}(x)$ is equivalent to the pendulum's spin and is spatially constant.

The above analysis shows that at late times, the spatial structure of the solution is very simple: every $\widetilde{\mathbf{P}}_{v}$ has a precession about an axis $\widetilde{\mathbf{m}}_{0}(x)$ with a frequency $\frac{M_{0}}{v}$, where $\widetilde{\mathbf{m}}_{0}(x)$ itself has a motion equivalent to that of a gyroscopic pendulum in an inhomogeneous magnetic field, keeping a fixed length, spin, and energy. This similarity to the gyroscopic pendulum solution, in a corotating frame, is, however, limited to the conserved quantities and a formal similarity in the equations of motion. The actual motion is different, because $\widetilde{\mathbf{B}}$ in this case has a nontrivial motion that is not necessarily much slower than that of $\widetilde{\mathbf{M}}_{n}$. These conserved quantities are formally identical to those in Ref. [65], but they have a slightly different interpretation. Unlike in the above, where exactly $0+1 \mathrm{D}$ or $1+0 \mathrm{D}$ was considered, ours are obtained under a steady-state approximation in $1+1 \mathrm{D}$ and need not be exactly conserved.

\section{Nature of the solution}

We now prove that the solution at late times, described by Eq. (8a), cannot be separable in position and velocity coordinates-i.e., it cannot be written in the form

$$
\widetilde{\mathbf{P}}_{v}=G_{v} \widetilde{\mathbf{S}}_{v}=G_{v}\left(\begin{array}{c}
f_{1}(x) h_{1}(v) \\
f_{2}(x) h_{2}(v) \\
f_{3}(x) h_{3}(v)
\end{array}\right)
$$

In our notation, the components of $\mathbf{S}_{v}$ in the rotated frame look like $\widetilde{\mathbf{S}}_{v}=\left(\widetilde{\boldsymbol{s}}_{v}^{(1)}, \widetilde{\boldsymbol{s}}_{v}^{(2)}, \widetilde{\boldsymbol{s}}_{v}^{(3)}\right)^{T}$, with $\left|\widetilde{\mathbf{S}}_{v}\right|=1$. For the above type of solution, clearly $f_{1}(x)=f_{2}(x)=f_{3}(x)$ is not possible. Otherwise, the normalization of $\widetilde{\mathbf{S}}_{v}$ for a fixed velocity mode will be different at different points in space, which is obviously unphysical. By a similar argument, $h_{1}(v)=h_{2}(v)=h_{3}(v)$ cannot be possible either, as that will lead to $\widetilde{\mathbf{S}}_{v}$ having different normalizations for different velocity modes at a fixed point in space. Plugging Eq. (21) into Eq. (8a), we get two separate sets of equations-one governing the spatial dependence, and the other with the velocity dependence of the full solution. The equations governing the velocity dependence look like

$$
\begin{aligned}
& v h_{1}(v)=H_{2} h_{3}(v)-h_{2}(v) H_{3}, \\
& v h_{2}(v)=H_{3} h_{1}(v)-h_{3}(v) H_{1}, \\
& v h_{3}(v)=H_{1} h_{2}(v)-h_{1}(v) H_{2},
\end{aligned}
$$

where

$$
\begin{aligned}
& H_{1}=\int_{-1}^{1} G_{v} h_{1}(v) d v, \\
& H_{2}=\int_{-1}^{1} G_{v} h_{2}(v) d v, \\
& H_{3}=\int_{-1}^{1} G_{v} h_{3}(v) d v .
\end{aligned}
$$

For all modes with $v \neq 0$, Eqs. (22a)-(22c) can be satisfied only if $h_{1}(v)=h_{2}(v)=h_{3}(v)=0$. This already suggests that, again, no meaningful solutions exist. However, we should confirm that the spatial solutions do not diverge. The equations for the spatial dependence look like

$$
\begin{aligned}
\frac{d}{d x} f_{1}(x) & =f_{2}(x) f_{3}(x), \\
\frac{d}{d x} f_{2}(x) & =f_{1}(x) f_{3}(x), \\
\frac{d}{d x} f_{3}(x) & =f_{1}(x) f_{2}(x) .
\end{aligned}
$$

To solve Eqs. (24a), (24b), and (24c), we multiply them by $f_{1}(x), f_{2}(x)$, and $f_{3}(x)$, respectively, which gives

$$
\begin{aligned}
\frac{d}{d x} \frac{f_{1}(x)^{2}}{2} & =\frac{d}{d x} \frac{f_{2}(x)^{2}}{2}=\frac{d}{d x} \frac{f_{3}(x)^{2}}{2} \\
& =f_{1}(x) f_{2}(x) f_{3}(x) .
\end{aligned}
$$

Equation (25) implies

$$
\begin{aligned}
& f_{1}(x)^{2}=f_{3}(x)^{2}+C_{1}, \\
& f_{2}(x)^{2}=f_{3}(x)^{2}+C_{2},
\end{aligned}
$$

where $C_{1}$ and $C_{2}$ are integration constants. The choices $C_{1}=C_{2}=0$ are disallowed, as that will mimic the case $f_{1}(x)=f_{2}(x)=f_{3}(x)$. Equations (26a) and (26b), along with Eq. (24c), give

$$
\frac{d}{d x} f_{3}(x)= \pm \sqrt{f_{3}(x)^{2}+C_{1}} \sqrt{f_{3}(x)^{2}+C_{2}} .
$$

Solving Eq. (27), one gets

$$
\frac{-i}{\sqrt{C_{2}}} F\left(\sin ^{-1}\left[\sqrt{-1 / C_{1}} f_{3}(x)\right], \frac{C_{1}}{C_{2}}\right)=x+C_{3},
$$

where $F\left(\sin ^{-1}\left[\sqrt{-1 / C_{1}} f_{3}(x)\right], \frac{C_{1}}{C_{2}}\right)$ is an elliptic integral of first kind defined as

$$
F(\phi, m)=\int_{0}^{\phi}\left(1-m \sin ^{2} \theta\right)^{-1 / 2} d \theta,
$$


and $C_{3}$ is an integration constant. One has to basically invert Eq. (28) to get the behavior of $f_{3}(x)$ as a function of $x$. This function is not infinite everywhere and concludes our analytical proof that there are no separable solutions in steady state.

Still, this may be opaque, and to give a flavor for the solution, consider a special case with $C_{1}=C_{2}$, and the solution for Eq. (27) then becomes

$$
f_{3}(x)= \pm C_{1} \tanh \left(\sqrt{C}_{1} x \pm \sqrt{C}_{1} C_{3}\right) .
$$

For any value of $C_{1}$ and $C_{3}$, the solutions for $f_{1}(x), f_{2}(x)$, and $f_{3}(x)$, are finite-with either oscillatory or constant behavior over all space. This implies that for all the modes with $v \neq 0$, the solution for all the components of $\widetilde{\mathbf{S}}_{v}(x)$ will be exactly zero, giving rise to an unphysical solution. Another qualitative way to understand this is from Eq. (4), where one can see that due to the spatial dependence of the velocity-dependent term, $\frac{\mathbf{M}_{0}(x)}{v} \times \mathbf{P}_{v}(x)$, on the right-hand side of the equation, it cannot be rotated away by a rotation of $\mathbf{P}_{v}(x)$ in the position space. So, the equation of motion governing the spatial behavior for each $\mathbf{P}_{v}(x)$ cannot be the same for every velocity mode $v$, indicating a nonseparable solution in $x$ and $v$.

\section{Approach to steady state}

In this section, we consider the approach to steady state, without making the steady-state approximation. Rather, we investigate how the spatially averaged polarization vectors rearrange themselves at late times, conserving lepton number and giving rise to flavor depolarization over a range of velocities.

\section{Dependence on lepton asymmetry}

Polarization vectors cannot be completely depolarized if the lepton asymmetry is nonvanishing. To see this, we integrate both sides of Eq. (2) over all velocity modes to get

$$
\partial_{x} \mathbf{M}_{1}(x, t)+\partial_{t} \mathbf{M}_{0}(x, t)=0 .
$$

Performing a spatial average on both sides of Eq. (31) over the entire box with the periodic boundary condition $\mathbf{M}_{1}(L, t)=\mathbf{M}_{1}(0, t)$, one gets

$$
d_{t}\left\langle\mathbf{M}_{0}(t)\right\rangle=0 .
$$

This implies

$$
\left\langle\mathbf{M}_{0}(t)\right\rangle=\text { constant },
$$

where $\left\langle\mathbf{M}_{0}(t)\right\rangle$ is understood as

$$
\left\langle\mathbf{M}_{0}(t)\right\rangle=\frac{1}{L} \int_{0}^{L} d x \mathbf{M}_{0}(x, t) .
$$

Using the approximate stationarity at late times, one can argue from Eq. (31) that $\mathbf{M}_{1}(x, t)$ is approximately constant all over space. The constant in Eq. (33) can be determined from the initial condition of the system, where all neutrinos are emitted as approximately flavor-pure states from every point in space-i.e., $s_{v}^{(3)}(x, 0)=1$ for all $x$ and $v$. One can write $\left\langle\mathbf{M}_{0}(t)\right\rangle$ as

$$
\left\langle\mathbf{M}_{0}(t)\right\rangle=\left(\begin{array}{c}
\int_{-1}^{1} d v G_{v}\left\langle s_{v}^{(1)}(t)\right\rangle \\
\int_{-1}^{1} d v G_{v}\left\langle s_{v}^{(2)}(t)\right\rangle \\
\int_{-1}^{1} d v G_{v}\left\langle s_{v}^{(3)}(t)\right\rangle
\end{array}\right),
$$

which remains true at all times. The above initial conditions, and defining $\int_{-1}^{1} G_{v} d v=A$, give rise to a set of three conditions that have to be satisfied even in the nonlinear regime:

$$
\begin{aligned}
& \int_{-1}^{1} d v G_{v}\left\langle s_{v}^{(1)}\right\rangle=\int_{-1}^{1} d v G_{v}\left\langle s_{v}^{(2)}\right\rangle=0 \\
& \int_{-1}^{1} d v G_{v}\left\langle s_{v}^{(3)}\right\rangle=A .
\end{aligned}
$$

One way that Eqs. (36a) and (36b) can be satisfied at late times for systems with zero lepton asymmetry-i.e., $A=0$ - is if $\left\langle s_{v}^{(1)}\right\rangle=\left\langle s_{v}^{(2)}\right\rangle=\left\langle s_{v}^{(3)}\right\rangle=0$ for every velocity mode $v$. This indicates flavor depolarization. However, for systems with $A \neq 0$, such a simple solution can exist if $\left\langle s_{v}^{(3)}\right\rangle=1$ for every $v$. This is possible only for an inert system where fast flavor conversion does not occur. Therefore, fast flavor conversion in the nonlinear regime can happen for $A \neq 0$, if $\left\langle s_{v}^{(3)}\right\rangle$ behaves in such a velocitydependent way that it preserves the value of the lepton asymmetry $A$. We will find, numerically, in the case of $A$ being $+v e$ (or $-v e$ ), that $\left\langle s_{v}^{(3)}\right\rangle$ will remain much closer to 1 for the modes with $+v e$ (or $-v e$ ) values of $G_{v}$.

\section{Fast depolarization}

Now we will show that a set of polarization vectors can undergo a "fast depolarization"-i.e., the system exhibits a "fast spectral split"-like evolution, similar to what appears in the foundational study by Raffelt and Smirnov [17,18], that leads to $s_{v}^{(3)}(x, t)$ flipping (and approximately vanishing) over a range of velocities, but constrained by conserved lepton numbers.

Consider Eq. (3). After averaging over space, we get

$$
\partial_{t}\left\langle\mathbf{P}_{v}(t)\right\rangle=\left\langle\mathbf{M}_{0}(t) \times \mathbf{P}_{v}(t)\right\rangle-v\left\langle\mathbf{M}_{1}(t) \times \mathbf{P}_{v}(t)\right\rangle .
$$

The averaging is over the cross product of the vectors, but because the polarization vectors vary very fast over space, 
one expects that the averaging factorizes over the cross product, which gives

$$
\partial_{t}\left\langle\mathbf{P}_{v}(t)\right\rangle=\left(\left\langle\mathbf{M}_{0}(t)\right\rangle-v\left\langle\mathbf{M}_{1}(t)\right\rangle\right) \times\left\langle\mathbf{P}_{v}(t)\right\rangle .
$$

By integrating the above equation over all velocities, one recovers that $\left\langle\mathbf{M}_{0}\right\rangle$ is time independent. However, multiplying the above equation with $v$ and then integrating over $v$ gives

$$
\partial_{t}\left\langle\mathbf{M}_{1}(t)\right\rangle=\left(\left\langle\mathbf{M}_{0}(t)\right\rangle+\langle\mathbf{X}(t)\rangle\right) \times\left\langle\mathbf{M}_{1}(t)\right\rangle,
$$

where we define a new vector,

$$
\langle\mathbf{X}(t)\rangle=\int_{-1}^{+1} d v v^{2}\langle\mathbf{P}(t)\rangle .
$$

Equation (38) ensures that each $\left\langle\mathbf{P}_{v}(t)\right\rangle$ precesses around its Hamiltonian:

$$
\left\langle\mathbf{H}_{v}(t)\right\rangle=\left\langle\mathbf{M}_{0}(t)\right\rangle-v\left\langle\mathbf{M}_{1}(t)\right\rangle .
$$

Initially, all polarization vectors are either aligned or antialigned to $\hat{\mathbf{e}}_{3}$, and over time $\left\langle\mathbf{M}_{1}(t)\right\rangle$ has dynamics, while the polarization vectors are dragged by $\left\langle\mathbf{H}_{v}(t)\right\rangle$.

We assume that the polarization vectors stay close to their Hamiltonians, and thus remain in the plane formed by $\left\langle\mathbf{M}_{0}(t)\right\rangle$ and $\left\langle\mathbf{M}_{1}(t)\right\rangle$, ignoring the precession around their Hamiltonian. The vector $\langle\mathbf{X}(t)\rangle$ can be decomposed as

$$
\langle\mathbf{X}(t)\rangle=\alpha(t)\left\langle\mathbf{M}_{0}(t)\right\rangle+\left\langle\mathbf{X}_{\perp}(t)\right\rangle
$$

which, upon insertion in Eq. (39), gives

$$
\partial_{t}\left\langle\mathbf{M}_{1}(t)\right\rangle=\left((1+\alpha(t))\left\langle\mathbf{M}_{0}(t)\right\rangle+\left\langle\mathbf{X}_{\perp}(t)\right\rangle\right) \times\left\langle\mathbf{M}_{1}(t)\right\rangle \text {. }
$$

Note that $\langle\mathbf{X}(t)\rangle$ typically has dynamics at the same frequency as $\left\langle\mathbf{M}_{1}(t)\right\rangle$. So $\left\langle\mathbf{M}_{1}(t)\right\rangle$, which has the dynamics of a gyroscopic pendulum, not only can precess around $\left\langle\mathbf{M}_{0}(t)\right\rangle$ but also can have bipolar nutations.

In a frame that corotates with the plane formed by $\left\langle\mathbf{M}_{0}(t)\right\rangle$ and $\left\langle\mathbf{M}_{1}(t)\right\rangle$, the Hamiltonian shifts by $-(1+\alpha(t))\left\langle\mathbf{M}_{0}(t)\right\rangle$ due to pure precession of $\left\langle\mathbf{M}_{1}(t)\right\rangle$. Focusing on the $\hat{\mathbf{e}}_{3}$ component of the Hamiltonian, we find that

$$
\left\langle\widetilde{\mathbf{H}}_{v}^{(3)}(t)\right\rangle=-\alpha(t)\left\langle\mathbf{M}_{0}^{(3)}(t)\right\rangle-v\left\langle\mathbf{M}_{1}^{(3)}(t)\right\rangle .
$$

The components of $\left\langle\mathbf{M}_{0}(t)\right\rangle$ and $\left\langle\mathbf{M}_{1}(t)\right\rangle$ along $\hat{\mathbf{e}}_{3}$ remain conserved if $\left\langle\mathbf{M}_{1}(t)\right\rangle$ has no nutation. In any case, in addition to Eq. (36b), we can define another useful lepton number,

$$
\left\langle\mathbf{M}_{1}^{(3)}(t)\right\rangle=\int_{-1}^{+1} d v v G_{v}\left\langle s_{v}^{(3)}\right\rangle=B(t) .
$$

$\left\langle\mathbf{M}_{0}^{(3)}(t)\right\rangle$ stays constant at $A$, while $\left\langle\mathbf{M}_{1}(t)\right\rangle$ may either precess, keeping $B(t)$ constant, or flip by changing $B(t)$ if a bipolar instability is triggered. The $\hat{\mathbf{e}}_{3}$ component of the corotating Hamiltonian is therefore

$$
\left\langle\widetilde{\mathbf{H}}_{v}^{(3)}(t)\right\rangle=-\alpha(t) A-v B(t) .
$$

Depending on how $\alpha(t)$ and $B(t)$ vary with time, the above can change its sign between some initial time and final time. The condition for such a sign flip to occur is

$$
\left(\alpha_{\text {ini }} A+v B_{\text {ini }}\right)\left(\alpha_{\text {fin }} A+v B_{\text {fin }}\right)<0,
$$

where $(\ldots)_{\text {ini,fin }}$ are at the beginning and end of the evolution. If the Hamiltonian for a velocity mode $v$ can change sign by fulfilling Eq. (47), and obeying the constraints on $A$ and $B$, then that velocity mode following its Hamiltonian may flip its sign as well.

So far, our discussion closely follows the discussion in Refs. $[17,18]$. There are a few subtle differences. Unlike in the case of bipolar swaps where only the lepton number $A$ needs to remain conserved, here one has two constraint equations-i.e., conservation of $A$ and conservation or flip of $B$, depending on whether nutations occur. Further, this derivation was for spatially averaged polarization vectors, $\left\langle\mathbf{P}_{v}(t)\right\rangle$, that do not necessarily maintain the same length as their unaveraged counterparts $\mathbf{P}_{v}(t)$. This point is quite crucial, as the astute reader will notice; otherwise, there is no obvious source of irreversibility: unlike in the bipolar spectral swap where that is provided by a decreasing $\mu$, the irreversibility is provided by the relative dephasing-i.e., kinematic decoherence - of the polarization vectors, which is responsible for the irreversibility of $\alpha(t)$. Further, with simple choices of $G_{v}$, the swapping function of the $\left\langle s_{v}^{(3)}\right\rangle$ cannot be a mere sign flip over a block of velocities, in general. Such a sign change across a crossing can preserve $A$, but unless this block of $G_{v}$ is antisymmetric in $v$, such a flip does not obey the constraint on $B$.

More detailed exploration of fast depolarization will be published separately, but here we note a main qualitative feature: over time, the $\left\langle s_{v}^{(3)}\right\rangle$ for a range of velocities-say, $v<0$ - become close to zero, even flipping their signs, but others stay close to their initial state, $\left\langle s_{v}^{(3)}\right\rangle=1$. Consider a simple case, where $A>0, B>0$ and $G_{v}$ has a single crossing at zero. It is possible that the modes with $v \approx+1$ do not flip sign or become small, whereas those with smaller $v$ either become very small or flip their signs (with changes in magnitude, as well). The converse, however, is not possible, because such a configuration cannot preserve $A$ and $B$. On the other hand, when $A<0$ and $B<0$, the 
modes with $v \approx-1$ cannot flip sign. We will see this pattern in our numerical calculations.

\section{NUMERICAL STUDY}

In the following subsections, we now discuss our own numerical strategy to solve the equations of motion, the late-time behavior of the solution, and their relation to the analytical claims made in Sec. II.

We developed a code for solving Eq. (2). In our code, we discretize each of the spatial directions as well as the velocities, considering $N_{x}$ spatial modes and $N_{v}$ velocity modes, to get a total of $3 N_{x} N_{v}$ coupled nonlinear ODEs, where the factor of 3 comes due to the three elements of each polarization vector. It solves these coupled nonlinear ODEs as a function of time using Python's ZVODE solver, which is a complex-valued variable-coefficient ordinary differential equation solver in Python which implements a backward differentiation formula for doing numerical integration. The spatial derivatives at each spatial point are computed using Python's SCIPY.FFTPACK.DIFF package, which uses the fast Fourier transform method for calculating derivatives.

For the computations shown in this paper, we choose a periodic boundary condition in space such that, for any time $t$ and any velocity mode $v$, our solution satisfies

$$
\mathbf{P}_{v}(x, t)=\mathbf{P}_{v}(x+L, t) .
$$

We choose initial conditions such that all the neutrinos, with any velocity, are emitted as purely electron flavored states at every point in space. To trigger the flavor evolution, a perturbation of $10^{-6}$ is used as an initial seed for the transverse components of the polarization vectors, for every velocity mode at the midpoint of the $1 \mathrm{D}$ box. This choice is arbitrary, but motivated by trying to seed all wavelength modes of the instability equally, as opposed to using, say, homogeneous initial conditions that favor a specific mode. We ensure that the size of the box $L$ and the maximum time $t_{\text {final }}$ up to which we solve the equations are such that $2 t_{\text {final }}<L$, as a result of which the mode with the largest velocity does not see the boundary of the box when emitted from the center of the box, where we seed the instability.

Specifically, we choose a 1D box of size $L=115$ and discretize it into $2^{12}$ spatial bins to solve Eq. (2) numerically up to a time of $t_{\text {final }}=50$. We discretize the ELN distributions into $2^{7}$ velocity modes, giving rise to a total of $3 \times 2^{12} \times 2^{7}=1572864$ coupled ODEs. These choices are optimized to obtain sufficient accuracy and precision, as we show in the Appendix. To show the dependence of our results on the ELN, we consider three qualitatively different continuous ELN distributions shown in Fig. 1. We choose these ELNs in such a way that they all have a zero crossing in their velocity distribution for fast flavor conversion to occur, but they have lepton asymmetries that are either zero,

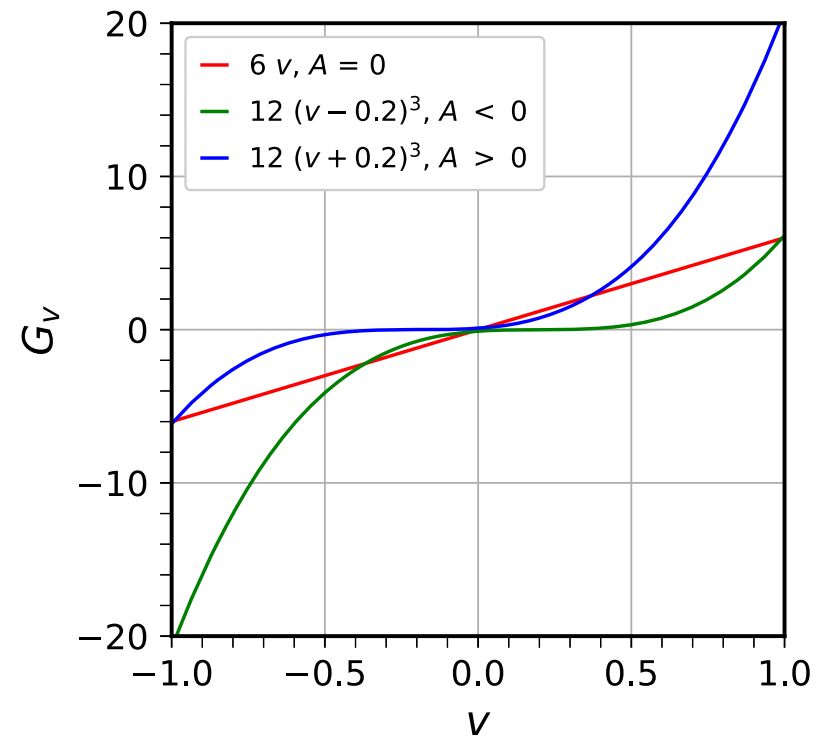

FIG. 1. ELN distributions used in our numerical examples. The case $A=0$ has vanishing lepton asymmetry, whereas $A<0$ and $A>0$ have more net negative and positive lepton numbers, respectively.

or negative, or positive. Figure 2 clearly indicates that the system becomes approximately steady already at $t_{\mathrm{NL}} \approx 10$, and to ensure that we are deeply in the nonlinear regime, we run our code until $t=50$.

We define some notation for convenience:

$$
\begin{aligned}
\mathbf{S}_{v}^{\perp}(x, t) & =s_{v}^{(1)}(x, t)-i s_{v}^{(2)}(x, t) \\
& =\left|\mathbf{S}_{v}^{\perp}(x, t)\right| e^{i \phi_{v}(x, t)},
\end{aligned}
$$

where $\phi_{v}(x, t)$ encodes the correlation between the two transverse components of the polarization vector for each velocity mode, with $\left|\mathbf{S}_{v}^{\perp}(x, t)\right|$ describing the length of the vector in the $\hat{\mathbf{e}}_{1}-\hat{\mathbf{e}}_{2}$ plane. We will denote all the spatially averaged quantities over the whole box of size $L$ as $\langle\cdots\rangle$, the velocity averaged quantities by $\cdots$, the probability distribution for the spatial distribution of some quantity as $\operatorname{PDF}(\cdots)$, and the standard deviation for that distribution as $\langle\langle\cdots\rangle\rangle$.

\section{A. Late-time behavior of parallel and perpendicular components}

In Fig. 3, we show the time evolution of the spatial average and standard deviation of $s_{v}^{(3)}(x, t)$ for all three types of lepton asymmetry. In Fig. 4, we show the histograms of $s_{v}^{(3)}(x, t)$ and $\left|\mathbf{S}_{v}^{\perp}(x, t)\right|$ at a time $t=50$ and for various velocity modes color coded by their velocity. We have checked that these histograms are themselves quasistationary at late times. These results indicate approximate stationarity in time in the nonlinear regime for all velocities. Note how $\left\langle s^{(3)}(t)\right\rangle$ separates into two cohorts, dictated 

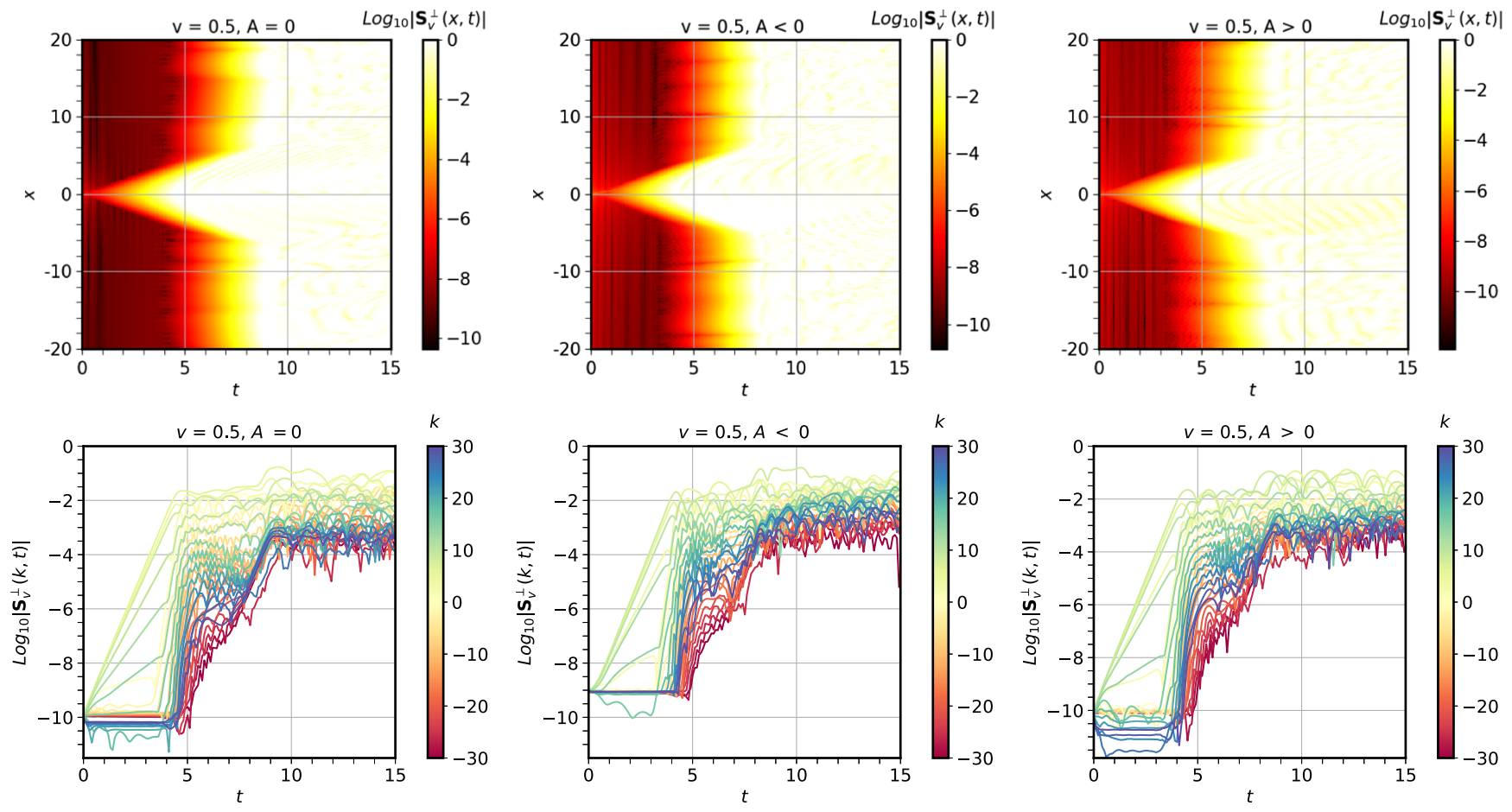

FIG. 2. Top: absolute value of the off-diagonal element of the density matrix, color coded as per the color bar, shown for the velocity mode $v=0.5$. Bottom: growth of the off-diagonal elements of the density matrix as a function of time for various different $k$ modes, color coded as per the color bar, for the same velocity mode. Left panels show the case $A=0$, middle panels $A<0$, and right panels $A>0$. Note the approximately steady-state solution at late times.
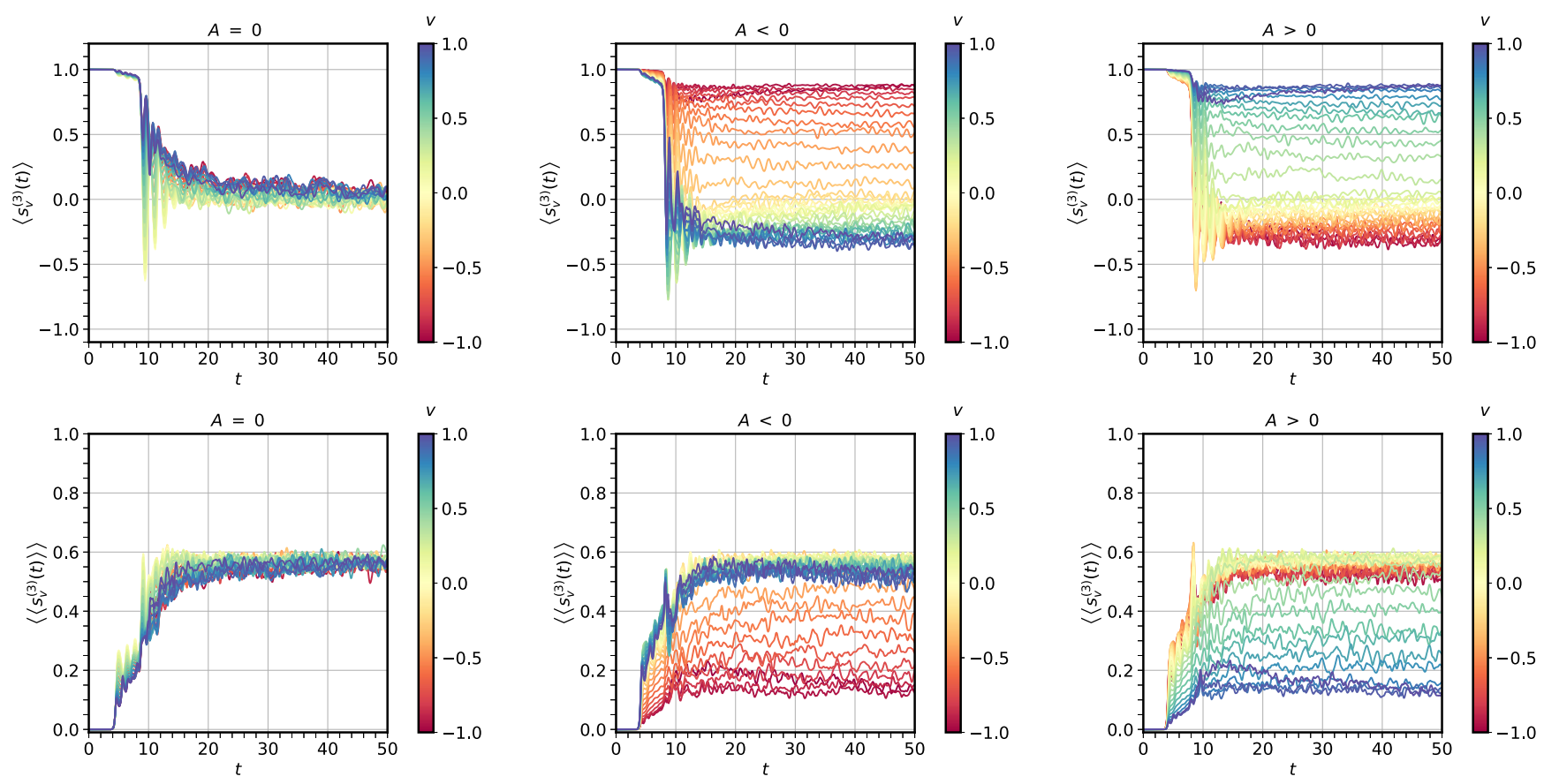

FIG. 3. Top: mean value of spatial distributions of $s_{v}^{(3)}$ varying with time, for a set of velocity modes color coded by the velocity. Bottom: standard deviation of the spatial distributions of $s_{v}^{(3)}$ as a function of time. Left panels show the case $A=0$, middle panels $A<0$, and right panels $A>0$. Note that both the spatial mean and standard deviation become approximately stationary in time in the nonlinear regime. Note also the spectral-swap-like separation of $s_{v}^{(3)}$ into two cohorts for $A \neq 0$. 

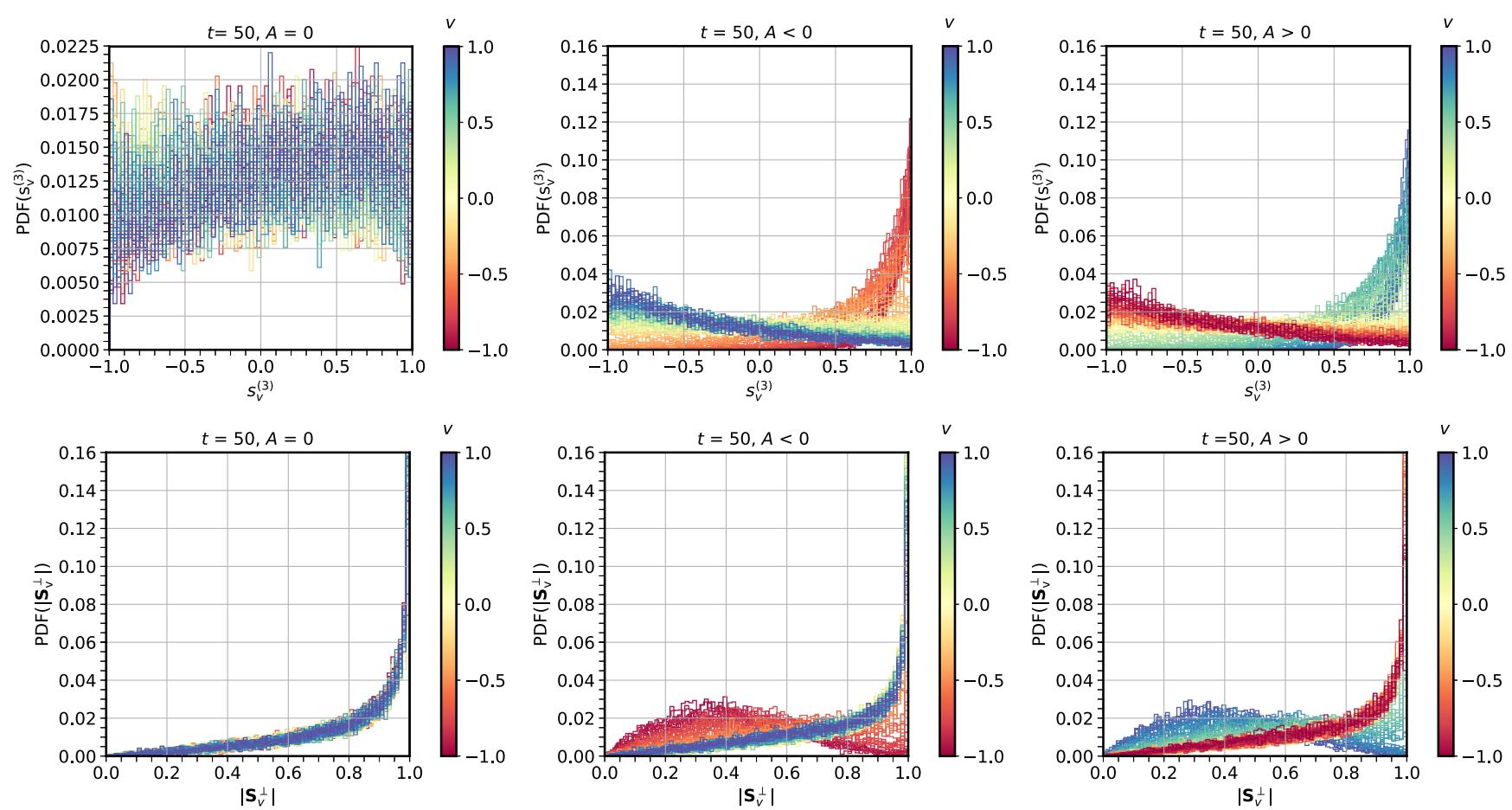

FIG. 4. Top: histograms of spatial distributions of $s_{v}^{(3)}$ at time $t=50$ for different velocity modes color coded by the velocity. Bottom: same for histograms for $\left|\mathbf{S}_{v}^{\perp}\right|$.
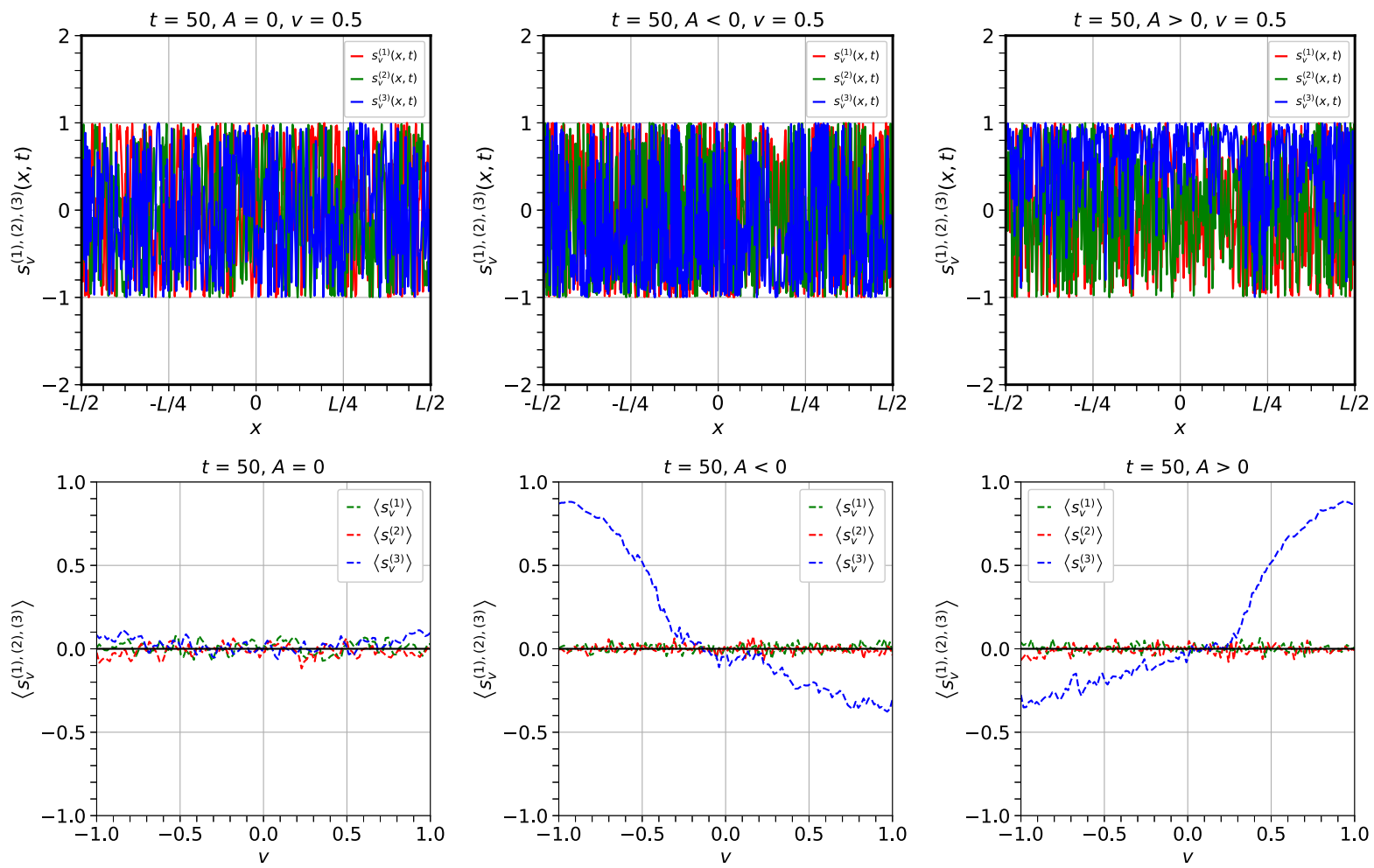

FIG. 5. Top: spatial variation of the three components of the polarization vector at time $t=50$ for $v=0.5$. Bottom: spatially averaged value of the three components of the polarization vector as a function of different velocity modes at time $t=50$. Left panels show the case $A=0$, middle panels $A<0$, and right panels $A>0$. Note the fast oscillations over spatial coordinates, but a relatively simpler velocity dependence for the spatially averaged solution. For $A=0$ there is flavor depolarization, whereas there is a partial depolarization, with an incomplete swap, for $A \neq 0$. 
essentially by the sign of $v$, at late times in the cases $A>0$ and $A<0$. This behavior is very similar to spectral swaps. As explained in Sec. II, which modes remain close to their initial state and which move away depends on $A, B$, and the higher moments.

The spatial distribution of $s_{v}^{(3)}$-i.e., $\operatorname{PDF}\left(s_{v}^{(3)}\right)$ - and the distribution of $\left|\mathbf{S}_{v}^{\perp}\right|$-i.e., $\operatorname{PDF}\left(\left|\mathbf{S}_{v}^{\perp}\right|\right)$ - shown in Fig. 4, are obviously related, because $\left|\mathbf{S}_{v}\right|=1$. The latter is obtained from the former by multiplying with the Jacobian of the transformation from $s_{v}^{(3)}$ to $\left|\mathbf{S}_{v}^{\perp}\right|$ :

$$
\operatorname{PDF}\left(\left|\mathbf{S}_{v}^{\perp}\right|\right)=\frac{\left|\mathbf{S}_{v}^{\perp}\right|}{\sqrt{1-\left|\mathbf{S}_{v}^{\perp}\right|^{2}}} \operatorname{PDF}\left(s_{v}^{(3)}\right) .
$$

Consider the case with $A=0$. In this case, $\operatorname{PDF}\left(s_{v}^{(3)}\right)$ is approximately uniform (see the top-left panel of Fig. 4), and as a result, $\operatorname{PDF}\left(\left|\mathbf{S}_{v}^{z_{\perp}}\right|\right)$ should be a sharply peaked distribution for every $v$ with a peak very close to $\left|\mathbf{S}_{v}^{\perp}\right|=1$ and a tail at $\left|\mathbf{S}_{v}^{\perp}\right|=0$. In the bottom-left panel of Fig. 4, we recover this unsurprising feature. For cases with $A<0$ $(A>0)$, the negative (positive) velocity modes show a peaked distribution in $s_{v}^{(3)}$ almost close to 1 , implying a more broadened distribution for $\left|\mathbf{S}_{v}^{\perp}\right|$ between 0 and 1, as seen in the middle and right panels.

The top panels of Fig. 5 show the oscillatory nature of the spatial variation of $s_{v}^{(3)}(x, t)$ at time $t=50$, when the system becomes fully nonlinear. The bottom panels of Fig. 5 show that the spatially averaged polarization vectors are depolarized for $A=0$, with $\left\langle s_{v}\right\rangle \approx 0$, but not so in the case of nonzero lepton asymmetry. For $A \neq 0$, the polarization vectors have not flipped close to $v \approx-1(v \approx+1)$ for $A<0(A>0)$, as predicted by our analysis of fast spectral swaps. One interesting point to note from the bottommiddle and bottom-right panels of Fig. 5, with spectra
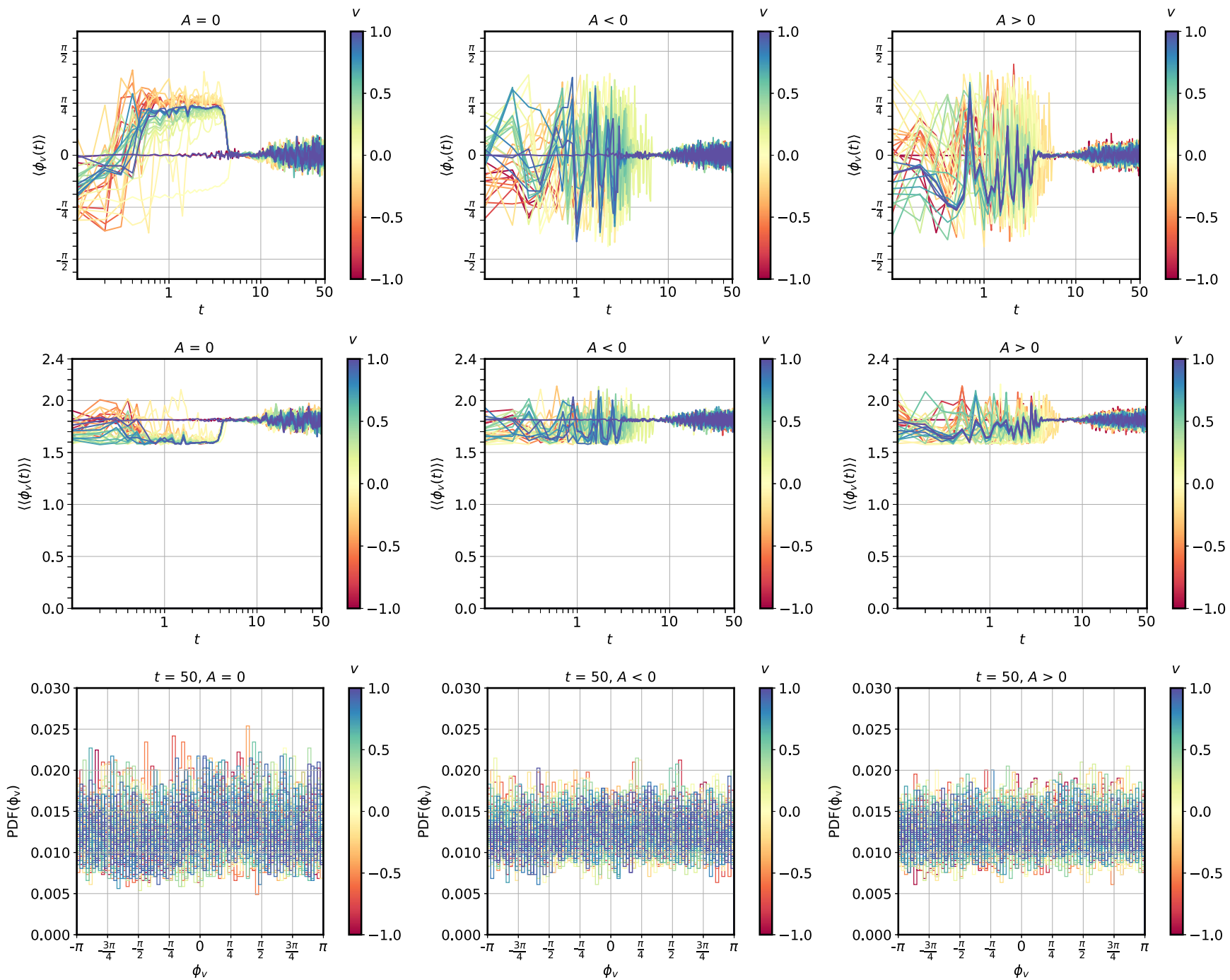

FIG. 6. Top: average of the spatial distribution of $\phi_{v}(x, t)$ at time $t=50$. Middle: standard deviations. Bottom: histograms of the spatial distribution of $\phi_{v}(x, t)$. In each plot, different velocity modes are depicted by color coding as per the color bar. Left panels show the case $A=0$, middle panels $A<0$, and right panels $A>0$. Note the approximate randomization of $\phi_{v}(x, t)$. 

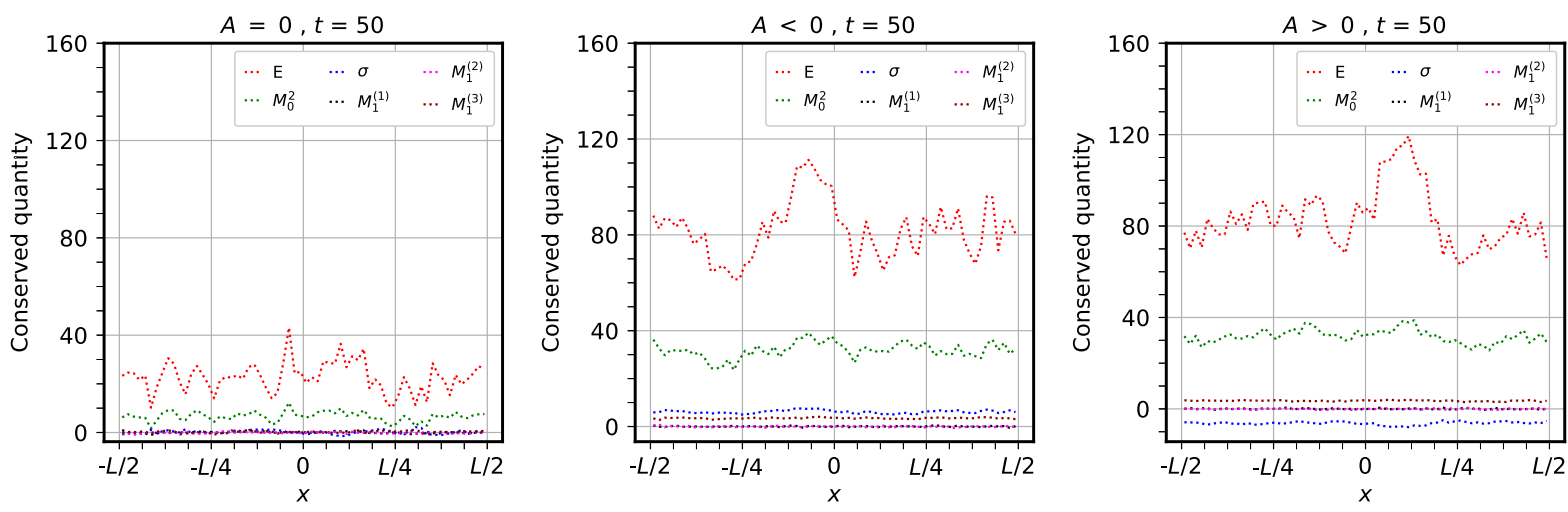

FIG. 7. Top: spatial variations of the conserved quantities $\left(\mathbf{M}_{1}, M_{0}^{2}, \sigma, E\right)$ that are supposed to be spatially constant in the extreme nonlinear regime. Left panels show the case $A=0$, middle panels $A<0$, and right panels $A>0$. We believe that the approximate-ness of the constancy of these conserved quantities is related to the approximate-ness of the steady state.

$A \neq 0$, is that the functional behavior in the velocity space shows a mirror symmetry when we switch the sign of $A$. This is not surprising, because our chosen ELNs have a symmetry $G_{A<0}(v)=-G_{A>0}(-v)$.

\section{B. Behavior of the phase $\phi_{v}(x, t)$}

We obtain the phase $\phi_{v}(x, t)$, for a given space-time point $(x, t)$ with a velocity $v$, in the range $[-\pi, \pi]$ using the Python package MATH.ATAN2, which takes $s_{v}^{(1)}(x, t)$ and $s_{v}^{(2)}(x, t)$ as inputs, obtained from our numerical simulation, and returns the principal value of $\arg \left(\mathbf{S}_{v}^{\perp}(x, t)\right)$ as the output.

Figure 6 shows that the phase is almost uniformly random, and its mean and variance are consistent with those expected of a uniformly random distribution over space, for every velocity mode and for any type of lepton asymmetry. Initially, for each velocity mode, the transverse components of a polarization vector $\mathbf{S}_{v}(x, t)$ are correlated, but in the extreme nonlinear regime, such a kinematic phase coherence is lost.

\section{Conserved quantities}

In Sec. II, we showed that $\mathbf{M}_{1}$ is almost constant in space in the steady-state approximation, which allowed us to go to a rotating frame where $\widetilde{\mathbf{M}}_{0}$ has a motion equivalent to a gyroscopic pendulum. Further, the pendulum's length $M_{0}$, spin $\sigma$, and energy $E$ can be seen to be spatially conserved. In this subsection, we undertake a numerical survey to verify if these quantities indeed satisfy the above analytical claim.

To calculate the different multipole moments numerically, we use the late-time solution for $\mathbf{P}_{v}(x, t)$ from our code and integrate over all the velocity modes from -1 to 1 at each spatial point with an appropriate weightage factor, corresponding to the Legendre polynomial of a degree equivalent to the multipole number of that particular moment. One technical issue is that the quantities $\sigma$ and
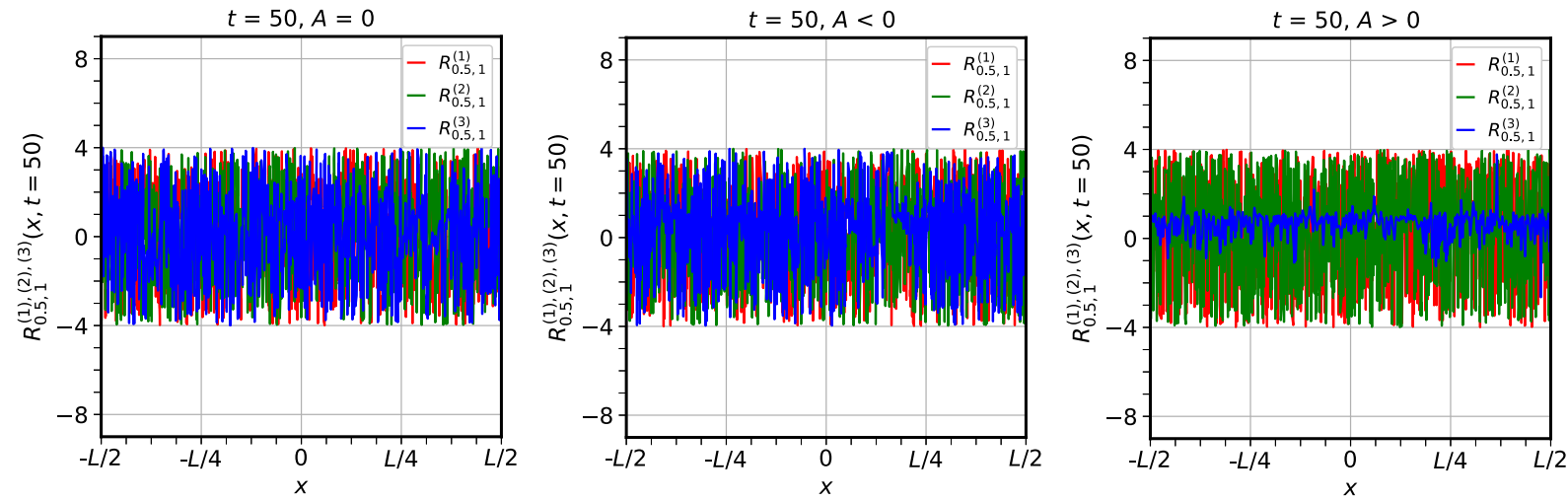

FIG. 8. Variation of $R_{v_{1}, v_{2}}^{(i)}(x, t)$ as a function of $x$ at $t=50$ is shown for $v_{1}=0.5, v_{2}=1$. The large variation as a function of $x$ shows that the late-time solution is not separable in space and velocity. In other words, at late times the different velocity modes do not show an approximately collective (correlated) evolution in space. 
$E$ involve infinite series in the multipole moments which are numerically impossible to calculate. Thankfully, the spatially averaged components of the polarization vectors, shown in the bottom panel of Fig. 5, behave very smoothly as a function of velocity and can be adequately approximated by the first few multipole moments.

Figure 7 shows the numerical results for the spatial variation of the conserved quantities at late times. Most of the conserved quantities are approximately constant over space, but the energy $E$ of the gyroscopic pendulum does not appear to be sufficiently constant (relative to, say, the variation of individual polarization vectors). Even at late times, the stationarity of the components of $\widetilde{\mathbf{S}}_{v}(x, t)$ is approximate, which is reflected in the degree of variation in the spatial behavior of the conserved quantities. In contrast, if one were to look at the velocity averaged $\overline{\mathbf{P}}(x, t)$, that is much noisier even at late times, not unlike what is shown in the upper panels of Fig. 5.

To verify if Eq. (2) has a separable solution for the components of $\mathbf{S}_{v}(x, t)$ in position and velocity space, we define a ratio as $R_{v_{1}, v_{2}}^{(i)}(x, t)=\frac{s_{v_{1}}^{(i)}(x, t)}{s_{v_{2}}^{(i)}(x, t)}$ for the $i$ th component of the polarization vector at a time $t$ in the full nonlinear regime for two different velocity modes $v_{1}, v_{2}$. This will be spatially constant if the solution is separable in $x$ and $v$. To calculate this quantity, we consider the solution at $t=50$ with the modes $v_{1}=0.5$ and $v_{2}=1$ for all three components $(i=1,2,3)$ of the polarization vector, and we plot $R_{v_{1}, v_{2}}^{(i)}(x, t)$ as a function of $x$. Figure 8 shows a large deviation from a constant, confirming a nonseparable solution in the nonlinear regime.

\section{CONCLUSIONS}

Our goal in this paper was to study the late-time behavior of fast oscillations. To this end, we considered a model in $1+1 \mathrm{D}$ and gave an analytical as well as a numerical understanding of its flavor dynamics. Here we summarize our main results:

(1) The system reaches an approximately steady state in time in the extreme nonlinear regime.

(2) In a rotating frame, the spatial evolution of all the polarization vectors is a velocity-dependent precession about a common axis.

(3) This common axis acts as a gyroscopic pendulum with a fixed length, spin, and energy, leading to an oscillatory behavior in position space.

(4) The steady-state solution for the components of the polarization vector is not separable in position and momentum space.

(5) The phase of the transverse polarization vector at different spatial locations becomes randomly distributed over the interval $[-\pi, \pi]$ at late times for all velocity modes and with any value of lepton asymmetry.
(6) The velocity dependence of the components of the polarization vector is controlled by the lepton asymmetry of the system. Systems with zero lepton asymmetry have a "decoherent" behavior for all velocity modes, with $\left\langle s_{v}^{(1)}\right\rangle=\left\langle s_{v}^{(2)}\right\rangle=\left\langle s_{v}^{(3)}\right\rangle=0$ for every $v$. In the case of nonzero lepton asymmetry, the decoherence is not complete, but $\left\langle s_{v}^{(1)}\right\rangle$ and $\left\langle s_{v}^{(2)}\right\rangle$ still remain zero for every velocity mode, while $\left\langle s_{v}^{(3)}\right\rangle$ shows some velocity dependence to conserve the lepton asymmetry.

(7) The behavior of $\left\langle s_{v}^{(3)}\right\rangle$ in the velocity space shows a mirror symmetry if the sign of the lepton asymmetry is flipped.

(8) $\left\langle s_{v}^{(3)}\right\rangle$ changes with time in a way reminiscent of spectral swaps. At late times, the configuration becomes steady and is given by a velocity-dependent "swap" function. The constraints on $A$ and $B$ must be obeyed for such a "swap."

We hope that these results provide some insight into the late-time flavor dynamics associated with fast flavor conversions of self-interacting neutrinos. Although we have chosen to study a simple system in $1+1 \mathrm{D}$, some of these physics results may be useful in understanding the more realistic scenario associated with neutrinos in a corecollapse supernova.

\section{ACKNOWLEDGMENTS}

This work is partially supported by the Department of Atomic Energy (Government of India) Research Project No. 12-R\&D-TFR5.02-0200, by the Department of Science and Technology (Government of India) through a Ramanujan Fellowship, and by the Max-PlanckGesellschaft through a Max Planck Partner Group awarded to B.D. The numerical computations were done on the Pride and Flock computing clusters in the Department of Theoretical Physics at TIFR Mumbai.

\section{APPENDIX: ERROR ESTIMATE}

In the left panel of Fig. 9, we illustrate the precision to be expected of our calculation. We solve the problem, outlined in the main text, for a sequence of increasingly fine discretizations for space $\left(N_{x}\right)$ and velocity $\left(N_{v}\right)$, with the $\log _{2}$ of the number of divisions noted in the legend of the figure. We check for convergence by comparing the results for these discretizations with the discretization $N_{x}=2^{12}$ and $N_{v}=2^{8}$. Our results indicate that a discretization of $N_{x}=2^{12}$ and $N_{v}=2^{7}$ is at most $\mathcal{O}\left(10^{-8}\right)$ off from yet finer discretizations.

In the right panel of Fig. 9, we show the accuracy expected of our calculation. We check if the lengths of the polarization vectors remain fixed at 1 . The error we incur on this is a lower bound on the error in our calculations. We find that our chosen discretization, $N_{x}=2^{12}$ and $N_{v}=2^{7}$, does as well as finer discretizations, making an error of 

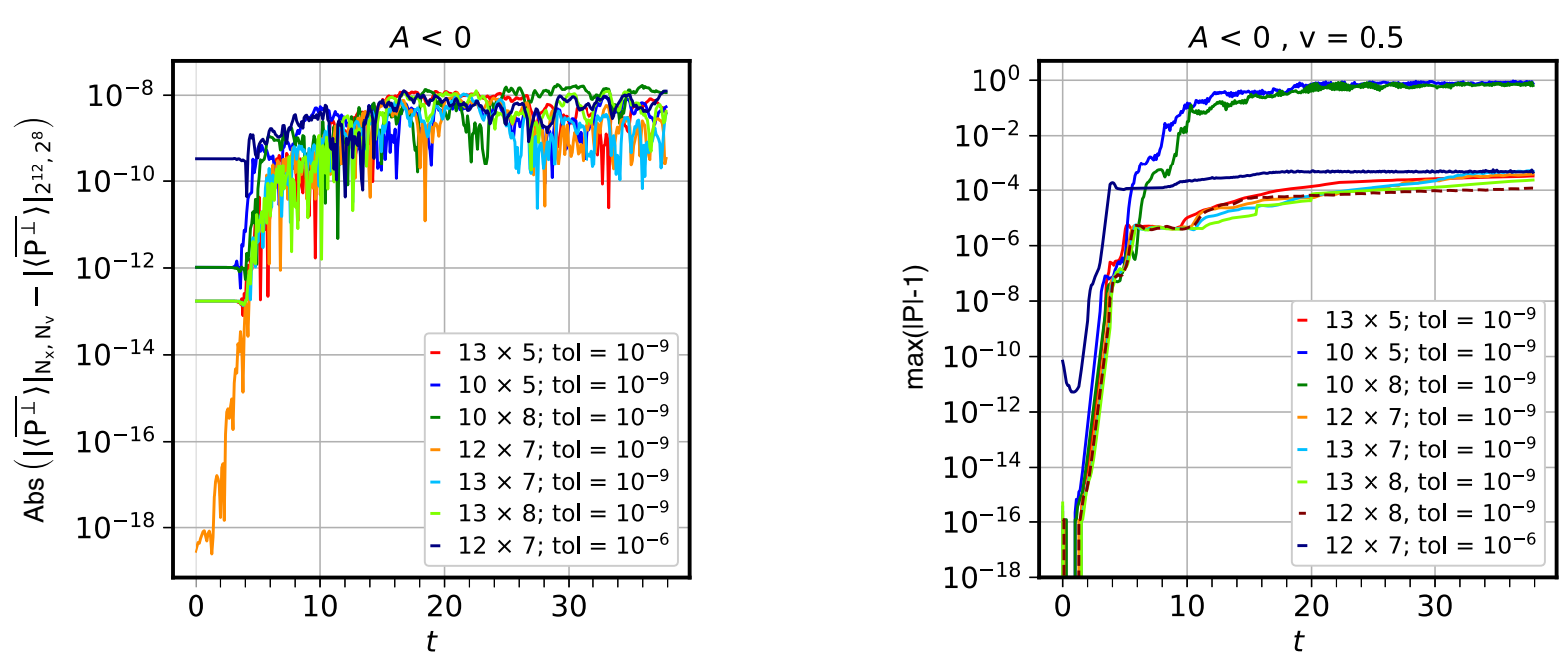

FIG. 9. Left: precision of our calculation, estimated by convergence of the computed $\left|\overline{\left\langle\mathbf{P}^{\perp}\right\rangle}\right|$ for different discretizations $N_{x} \times N_{v}$ (to be read as the exponents of 2-i.e., $12 \times 7$ refers to $N_{x}=2^{12}$ and $N_{v}=2^{7}$ ), as a function of time $t$, shown for the $A<0$ case. Right: accuracy of our calculation, estimated by maximum departure (over all space) of the magnitude of $\mathbf{P}_{v=0.5}$ from unity, as a function of time $t$, shown for the $A<0$ case and different discretizations. Absolute and relative tolerances were taken to be same and are mentioned alongside.

$\mathcal{O}\left(10^{-4}\right)$ at late times. Considering that the quantities we are interested in are close to $\mathcal{O}(1)$, this error is tolerable. Also, we find that the tolerance dictates how fast the errors increase initially, but the errors plateau out at $\sim 100$ times the tolerance. Whereas, if one is interested in a solution that is accurate to $\mathcal{O}\left(10^{-4}\right)$, it is less expensive to raise the tolerance to $10^{-6}$, without a significant penalty on accuracy and with a significant gain in speed.
[1] G. G. Raffelt, Stars as Laboratories for Fundamental Physics (University Press, Chicago, 1996), p. 664.

[2] L. Wolfenstein, Neutrino oscillations in matter, Phys. Rev. D 17, 2369 (1978).

[3] S. P. Mikheev and A. Yu. Smirnov, Neutrino oscillations in an inhomogeneous medium: Adiabatic regime, Zh. Eksp. Teor. Fiz. 92, 404 (1987) [Sov. Phys. JETP 65, 230 (1987)], https://inspirehep.net/literature/254687.

[4] S. P. Mikheev and A. Yu. Smirnov, Resonance oscillations of neutrinos in matter, Usp. Fiz. Nauk. 153, 3 (1987) [Sov. Phys. Usp. 30, 759 (1987)].

[5] A. S. Dighe and A. Yu. Smirnov, Identifying the neutrino mass spectrum from the neutrino burst from a supernova, Phys. Rev. D 62, 033007 (2000).

[6] B. Dasgupta and A. Dighe, Phase effects in neutrino conversions during a supernova shock wave, Phys. Rev. D 75, 093002 (2007).

[7] G. L. Fogli, E. Lisi, A. Mirizzi, and D. Montanino, Damping of supernova neutrino transitions in stochastic shock-wave density profiles, J. Cosmol. Astropart. Phys. 06 (2006) 012.

[8] A. Friedland and A. Gruzinov, Neutrino signatures of supernova turbulence, arXiv:astro-ph/0607244.

[9] J. T. Pantaleone, Dirac neutrinos in dense matter, Phys. Rev. D 46, 510 (1992).
[10] J. T. Pantaleone, Neutrino oscillations at high densities, Phys. Lett. B 287, 128 (1992).

[11] V. A. Kostelecky and S. Samuel, Selfmaintained coherent oscillations in dense neutrino gases, Phys. Rev. D 52, 621 (1995).

[12] S. Pastor, G. G. Raffelt, and D. V. Semikoz, Physics of synchronized neutrino oscillations caused by selfinteractions, Phys. Rev. D 65, 053011 (2002).

[13] H. Duan, G. M. Fuller, and Y.-Z. Qian, Collective neutrino flavor transformation in supernovae, Phys. Rev. D 74, 123004 (2006).

[14] H. Duan, G. M. Fuller, J. Carlson, and Y.-Z. Qian, Simulation of coherent non-linear neutrino flavor transformation in the supernova environment: 1. Correlated neutrino trajectories, Phys. Rev. D 74, 105014 (2006).

[15] S. Hannestad, G. G. Raffelt, G. Sigl, and Y. Y. Y. Wong, Self-induced conversion in dense neutrino gases: Pendulum in flavour space, Phys. Rev. D 74, 105010 (2006); Erratum, Phys. Rev. D 76, 029901 (2007).

[16] L. Johns and G. M. Fuller, Strange mechanics of the neutrino flavor pendulum, Phys. Rev. D 97, 023020 (2018).

[17] G. G. Raffelt and A. Yu. Smirnov, Self-induced spectral splits in supernova neutrino fluxes, Phys. Rev. D 76, 081301 (2007); Erratum, Phys. Rev. D 77, 029903 (2008). 
[18] G. G. Raffelt and A. Yu. Smirnov, Adiabaticity and spectral splits in collective neutrino transformations, Phys. Rev. D 76, 125008 (2007).

[19] G. L. Fogli, E. Lisi, A. Marrone, and A. Mirizzi, Collective neutrino flavor transitions in supernovae and the role of trajectory averaging, J. Cosmol. Astropart. Phys. 12 (2007) 010 .

[20] B. Dasgupta, A. Dighe, G. G. Raffelt, and A. Yu. Smirnov, Multiple Spectral Splits of Supernova Neutrinos, Phys. Rev. Lett. 103, 051105 (2009).

[21] B. Dasgupta and A. Dighe, Collective three-flavor oscillations of supernova neutrinos, Phys. Rev. D 77, 113002 (2008).

[22] H. Duan, G. M. Fuller, and Y.-Z. Qian, Stepwise spectral swapping with three neutrino flavors, Phys. Rev. D 77, 085016 (2008).

[23] B. Dasgupta, A. Dighe, A. Mirizzi, and G. G. Raffelt, Spectral split in prompt supernova neutrino burst: Analytic three-flavor treatment, Phys. Rev. D 77, 113007 (2008).

[24] C. Doering, R. S. L. Hansen, and M. Lindner, Stability of three neutrino flavor conversion in supernovae, J. Cosmol. Astropart. Phys. 08 (2019) 003.

[25] A. Esteban-Pretel, S. Pastor, R. Tomas, G. G. Raffelt, and G. Sigl, Multi-angle effects in collective supernova neutrino oscillations, J. Phys. Conf. Ser. 120, 052021 (2008).

[26] H. Duan and A. Friedland, Self-Induced Suppression of Collective Neutrino Oscillations in a Supernova, Phys. Rev. Lett. 106, 091101 (2011).

[27] S. Chakraborty, T. Fischer, A. Mirizzi, N. Saviano, and R. Tomas, Analysis of matter suppression in collective neutrino oscillations during the supernova accretion phase, Phys. Rev. D 84, 025002 (2011).

[28] N. Saviano, S. Chakraborty, T. Fischer, and A. Mirizzi, Stability analysis of collective neutrino oscillations in the supernova accretion phase with realistic energy and angle distributions, Phys. Rev. D 85, 113002 (2012).

[29] G. G. Raffelt and G. Sigl, Self-induced decoherence in dense neutrino gases, Phys. Rev. D 75, 083002 (2007).

[30] A. Esteban-Pretel, S. Pastor, R. Tomas, Georg G. Raffelt, and G. Sigl, Decoherence in supernova neutrino transformations suppressed by deleptonization, Phys. Rev. D 76, 125018 (2007).

[31] R. S. L. Hansen and A. Yu. Smirnov, Neutrino conversion in a neutrino flux: Towards an effective theory of collective oscillations, J. Cosmol. Astropart. Phys. 04 (2018) 057.

[32] R. S. L. Hansen and A. Yu. Smirnov, Effect of extended $\nu$ production region on collective oscillations in supernovae, J. Cosmol. Astropart. Phys. 10 (2019) 027.

[33] A. Banerjee, A. Dighe, and G. Raffelt, Linearized flavorstability analysis of dense neutrino streams, Phys. Rev. D 84, 053013 (2011).

[34] G. Raffelt and D. de Sousa Seixas, Neutrino flavor pendulum in both mass hierarchies, Phys. Rev. D 88, 045031 (2013).

[35] G. Raffelt, S. Sarikas, and D. de Sousa Seixas, Axial Symmetry Breaking in Self-Induced Flavor Conversion of Supernova Neutrino Fluxes, Phys. Rev. Lett. 111, 091101 (2013); Erratum, Phys. Rev. Lett. 113, 239903 (2014).

[36] G. Mangano, A. Mirizzi, and N. Saviano, Damping the neutrino flavor pendulum by breaking homogeneity, Phys. Rev. D 89, 073017 (2014).
[37] H. Duan and S. Shalgar, Flavor instabilities in the neutrino line model, Phys. Lett. B 747, 139 (2015).

[38] S. Abbar, H. Duan, and S. Shalgar, Flavor instabilities in the multiangle neutrino line model, Phys. Rev. D 92, 065019 (2015).

[39] B. Dasgupta and A. Mirizzi, Temporal instability enables neutrino flavor conversions deep inside supernovae, Phys. Rev. D 92, 125030 (2015).

[40] H. Duan, G. M. Fuller, and Y.-Z. Qian, Collective neutrino oscillations, Annu. Rev. Nucl. Part. Sci. 60, 569 (2010).

[41] A. Mirizzi, I. Tamborra, H.-T. Janka, N. Saviano, K. Scholberg, R. Bollig, L. Hudepohl, and S. Chakraborty, Supernova neutrinos: Production, oscillations and detection, Riv. Nuovo Cimento 39, 1 (2016).

[42] S. Chakraborty, R. Hansen, I. Izaguirre, and G. Raffelt, Collective neutrino flavor conversion: Recent developments, Nucl. Phys. B908, 366 (2016).

[43] S. Horiuchi and J. P. Kneller, What can be learned from a future supernova neutrino detection?, J. Phys. G 45, 043002 (2018).

[44] R. F. Sawyer, Speed-up of neutrino transformations in a supernova environment, Phys. Rev. D 72, 045003 (2005).

[45] R. F. Sawyer, Neutrino Cloud Instabilities Just Above the Neutrino Sphere of a Supernova, Phys. Rev. Lett. 116, 081101 (2016).

[46] S. Chakraborty, R. S. Hansen, I. Izaguirre, and G. Raffelt, Self-induced neutrino flavor conversion without flavor mixing, J. Cosmol. Astropart. Phys. 03 (2016) 042.

[47] B. Dasgupta, A. Mirizzi, and M. Sen, Fast neutrino flavor conversions near the supernova core with realistic flavordependent angular distributions, J. Cosmol. Astropart. Phys. 02 (2017) 019.

[48] I. Izaguirre, G. Raffelt, and I. Tamborra, Fast Pairwise Conversion of Supernova Neutrinos: A Dispersion-Relation Approach, Phys. Rev. Lett. 118, 021101 (2017).

[49] F. Capozzi, B. Dasgupta, E. Lisi, A. Marrone, and A. Mirizzi, Fast flavor conversions of supernova neutrinos: Classifying instabilities via dispersion relations, Phys. Rev. D 96, 043016 (2017).

[50] A. Das, A. Dighe, and M. Sen, New effects of non-standard self-interactions of neutrinos in a supernova, J. Cosmol. Astropart. Phys. 05 (2017) 051.

[51] B. Dasgupta and M. Sen, Fast neutrino flavor conversion as oscillations in a quartic potential, Phys. Rev. D 97, 023017 (2018).

[52] A. Dighe and M. Sen, Nonstandard neutrino selfinteractions in a supernova and fast flavor conversions, Phys. Rev. D 97, 043011 (2018).

[53] S. Abbar and H. Duan, Fast neutrino flavor conversion: Roles of dense matter and spectrum crossing, Phys. Rev. D 98, 043014 (2018).

[54] T. Morinaga and S. Yamada, Linear stability analysis of collective neutrino oscillations without spurious modes, Phys. Rev. D 97, 023024 (2018).

[55] F. Capozzi, B. Dasgupta, and A. Mirizzi, Model-independent diagnostic of self-induced spectral equalization versus ordinary matter effects in supernova neutrinos, Phys. Rev. D 98, 063013 (2018). 
[56] B. Dasgupta, A. Mirizzi, and M. Sen, Simple method of diagnosing fast flavor conversions of supernova neutrinos, Phys. Rev. D 98, 103001 (2018).

[57] F. Capozzi, B. Dasgupta, A. Mirizzi, M. Sen, and G. Sigl, Collisional Triggering of Fast Flavor Conversions of Supernova Neutrinos, Phys. Rev. Lett. 122, 091101 (2019).

[58] S. Airen, F. Capozzi, S. Chakraborty, B. Dasgupta, G. Raffelt, and T. Stirner, Normal-mode analysis for collective neutrino oscillations, J. Cosmol. Astropart. Phys. 12 (2018) 019.

[59] S. Abbar and M. C. Volpe, On fast neutrino flavor conversion modes in the nonlinear regime, Phys. Lett. B 790 , 545 (2019).

[60] C. Yi, L. Ma, J. D. Martin, and H. Duan, Dispersion relation of the fast neutrino oscillation wave, Phys. Rev. D 99, 063005 (2019).

[61] J. D. Martin, S. Abbar, and H. Duan, Nonlinear flavor development of a two-dimensional neutrino gas, Phys. Rev. D 100, 023016 (2019).

[62] F. Capozzi, G. Raffelt, and T. Stirner, Fast neutrino flavor conversion: Collective motion vs. decoherence, J. Cosmol. Astropart. Phys. 09 (2019) 002.

[63] J. D. Martin, C. Yi, and H. Duan, Dynamic fast flavor oscillation waves in dense neutrino gases, Phys. Lett. B 800, 135088 (2020).

[64] M. Chakraborty and S. Chakraborty, Three flavor neutrino conversions in supernovae: Slow \& fast instabilities, J. Cosmol. Astropart. Phys. 01 (2020) 005.

[65] L. Johns, H. Nagakura, G. M. Fuller, and A. Burrows, Neutrino oscillations in supernovae: Angular moments and fast instabilities, Phys. Rev. D 101, 043009 (2020).

[66] S. Shalgar, I. Padilla-Gay, and I. Tamborra, Neutrino propagation hinders fast pairwise flavor conversions, J. Cosmol. Astropart. Phys. 06 (2020) 048.

[67] J. F. Cherry, G. M. Fuller, S. Horiuchi, K. Kotake, T. Takiwaki, and T. Fischer, Time of flight and supernova progenitor effects on the neutrino halo, Phys. Rev. D 102, 023022 (2020).

[68] S. Abbar, Searching for fast neutrino flavor conversion modes in core-collapse supernova simulations, J. Cosmol. Astropart. Phys. 05 (2020) 027.

[69] I. Tamborra, L. Huedepohl, G. Raffelt, and H.-T. Janka, Flavor-dependent neutrino angular distribution in corecollapse supernovae, Astrophys. J. 839, 132 (2017).

[70] M. Delfan Azari, S. Yamada, T. Morinaga, W. Iwakami, H. Okawa, H. Nagakura, and K. Sumiyoshi, Linear analysis of fast-pairwise collective neutrino oscillations in corecollapse supernovae based on the results of Boltzmann simulations, Phys. Rev. D 99, 103011 (2019).

[71] T. Morinaga, H. Nagakura, C. Kato, and S. Yamada, Fast neutrino-flavor conversion in the preshock region of core-collapse supernovae, Phys. Rev. Research 2, 012046 (2020).

[72] H. Nagakura, T. Morinaga, C. Kato, and S. Yamada, Fastpairwise collective neutrino oscillations associated with asymmetric neutrino emissions in core-collapse supernova, Astrophys. J. 886, 139 (2019).

[73] M. Delfan Azari, S. Yamada, T. Morinaga, H. Nagakura, S. Furusawa, A. Harada, H. Okawa, W. Iwakami, and K. Sumiyoshi, Fast collective neutrino oscillations inside the neutrino sphere in core-collapse supernovae, Phys. Rev. D 101, 023018 (2020).

[74] S. Abbar, H. Duan, K. Sumiyoshi, T. Takiwaki, and M. C. Volpe, Fast neutrino flavor conversion modes in multidimensional core-collapse supernova models: The role of the asymmetric neutrino distributions, Phys. Rev. D 101, 043016 (2020).

[75] R. Glas, H. T. Janka, F. Capozzi, M. Sen, B. Dasgupta, A. Mirizzi, and G. Sigl, Fast neutrino flavor instability in the neutron-star convection layer of three-dimensional supernova models, Phys. Rev. D 101, 063001 (2020). 